\title{
The design and implementation of a simulation platform for the running of high-speed trains based on High Level Architecture
}

\author{
X. Lin, Q. Y. Wang, Z. C. Liang \& X. Y. Feng \\ Southwest Jiaotong University, China
}

\begin{abstract}
In order to overcome the problems existing in the design and field testing of the Train's Auxiliary Operation System (TAOS), such as a complex environment, great losses, low efficiency and high risks, this paper describes an integrated driving simulation system for the high-speed train based on High Level Architecture (HLA). The simulation system consists of several simulation modules, including a signal system, traction power supply, train operation simulation and the actual driver control interface device equipped with virtual scene display. The train's running environment is simulated and implemented in the signal simulation module and traction power supply simulation module. The train's on-board equipment is simulated in the train operation simulation module. The driver control device offers the display interface of TAOS. As the simulator is composed of multiple complex sub-modules, the simulation step is different from each other. To test the efficiency of the train's auxiliary operation system, the whole system's real-time performance is very important. So the distributed simulation platform based on HLA is built. And the sub-modules are integrated based on RTI (Run-Time Infrastructure). Data transmission between the subsystems is also introduced in this paper.
\end{abstract}

Keywords: TAOS, high-speed train, HLA, distributed simulation platform, simulation module, RT.

\section{Introduction}

With the rapid development of China high-speed railway, the security operation of high-speed railway has been paid more and more attention. The mature driving experience of a driver guarantees the safe operation of a train. However, it's dangerous to train a new driver in the real vehicle as well as a waste of railway 
resource. In order to realize the train operation simulation in the lab, some researches about the train operation simulation have been done. For example, [1-3] respectively proposes the train driving simulation system based on TCP/IP, which makes the synchronous control between the subsystems in the train come true. A train bidirectional traction calculation unit model and the virtual operation scene of a train are put forward in [4]. Song and Zhang [5] have designed a kind of distributed three-dimensional scene simulator of a train operation based on High Level Architecture (HLA). And the simulator can access CTCS3 (China Train Control System) system. These papers above have realized the train operation simulation in the lab. However, more work should be done to make the train operation simulation closer to reality. For example, the power supply system and signal system must be taken into consideration. In this paper, an integrated train operation simulation platform combining the actual driver control interface device with the virtual running environment of a train has been put forward, which can be served as the basis research platform of the Train's Auxiliary Operation System (TAOS).

As shown in Fig. 1, the distributed simulation platform consists of traction net, signal system, AC drive module, braking module, CCU, ATP, traction calculation, $3 \mathrm{D}$ visualization and driver control interface device. Among them, there are three sub-modules including DMI, MMI and DCD (driver control device). To solve the problem of all sub-systems' synchronization management and monitor the operation status of every sub-module, the master station sub-module (not displayed in Fig. 1) is added in the simulation platform. AC drive module, braking module, CCU, ATP, traction calculation, DMI, MMI, DCD are train's on-board equipment. Then the dynamic simulation of the train's running can be realized. And the traction net is the power source of the train's running, coupling with the $\mathrm{AC}$ drive module. The signal module realizes the data transmission between a train and the rail circuit, balise. Meantime, 3D visualization can simulate the train's external running environment, which gives the driver real operation experience. In the process of simulation, 3D visualization receives the train's position and speed from the traction calculation module. What's more, the information of the train's position and speed can be displayed dynamically in 3D visualization. On basis of the distributed simulation platform, we can test the efficiency of the train's auxiliary operation system. Since the whole system's real-time performance is of great importance, the distributed simulation platform based on HLA is built with the sub-modules integrated based on RTI.

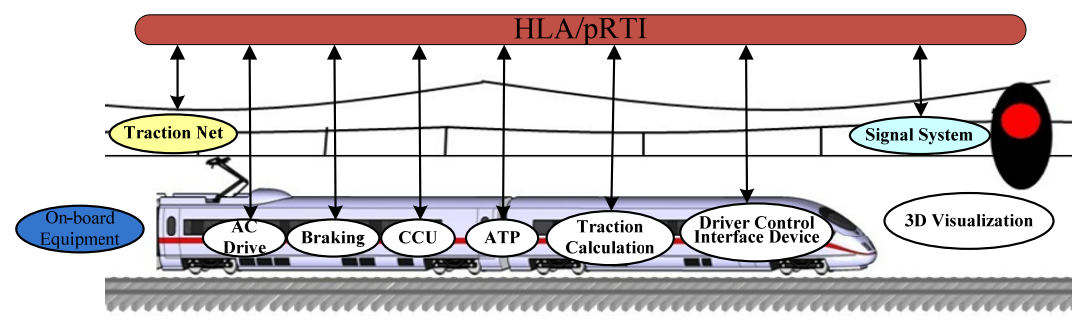

Figure 1: Distributed simulation platform of the train's running. 


\section{HLA}

\subsection{Brief introduction}

HLA (High Level Architecture) is a new distributed simulation framework which is proposed by US Department of Defense [6]. It can offer common and relatively independent RTI. And the function of the application layer and underlying infrastructure is separated by HLA. HLA only offers the communication interface. And the users don't need to concern about the underlying communication of HLA but pay more attention to the professional application development.

\subsection{Federate Process and Simulation Data Exchange}

In the HLA, federation is regarded as a distributed simulation system which achieves a particular purpose. It consists of many federates that can influence each other. For a single federate, its federate process is shown in Fig. 2.

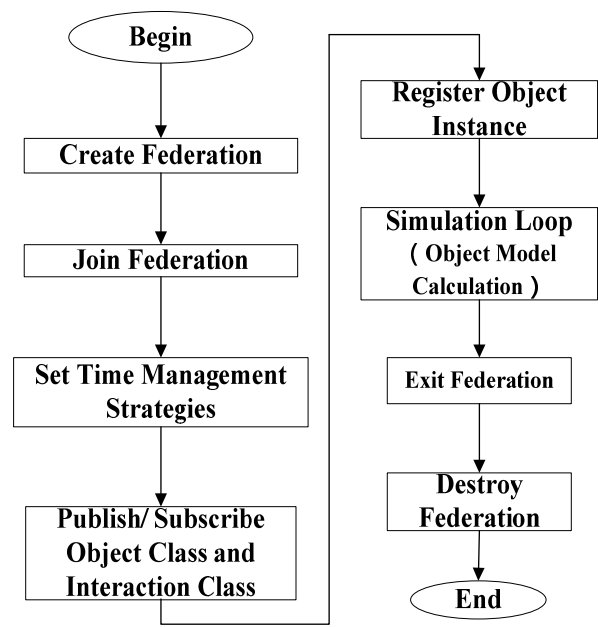

Figure 2: Federate process.

In this paper, Pitch Run-Time Infrastructure (RTI) which is developed by PITCH COMPANY in Sweden is used as a simulation tool to realize data exchange between modules. pRTI1516 is the run-time infrastructure of Pitch RTI based on IEEE1516 [7].

For example, MultiTrainSim is a federate. The file-MultiTrainSim.xml decides the number of federate and data interface between federates. pRTI1516 and its federates are displayed in Fig. 3. Fig. 3 shows all sub-modules during the simulation. And the master station sub-module joins in the federation as a federate. It is responsible for federation's synchronization management and monitoring the running status of every federate. For the sake of limitation of number of computers in the lab, part modules are in the same computer. 


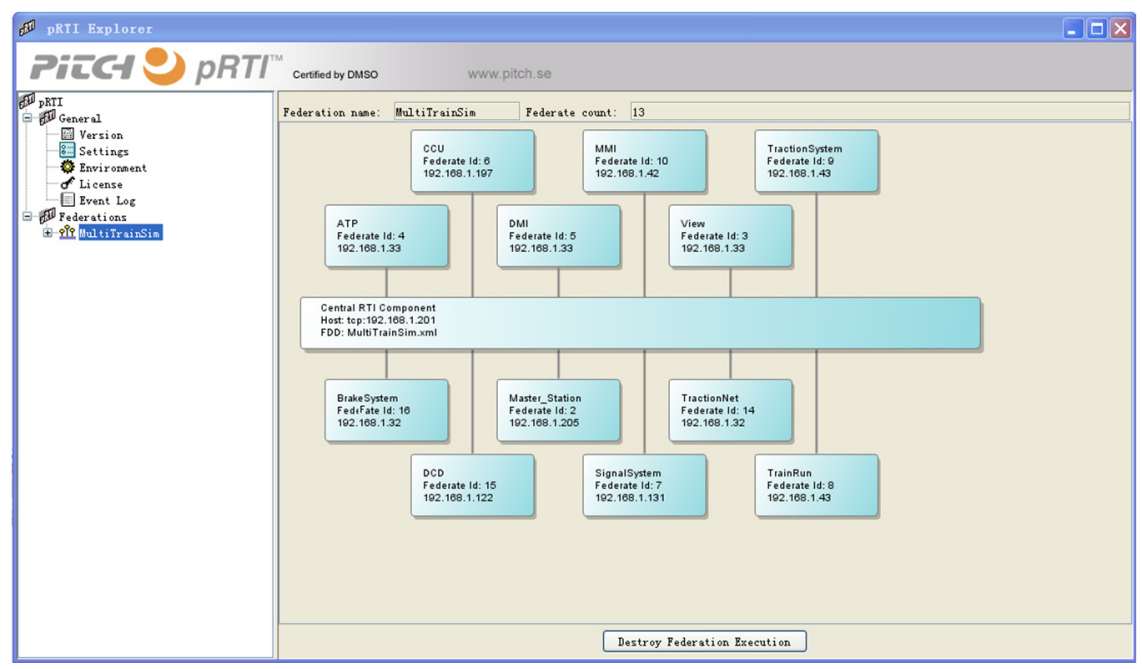

Figure 3: The Running Interface of pRTI1516.

To explain the progress of simulation data exchange, taking the data exchange between train calculation (i.e. TrainRun in Fig. 3) and 3D visualization (i.e. view in Fig. 3) as an example, the progress of simulation data exchange is analysed as follows.

The interactive interface between TrainRun and 3D visualization is defined below:

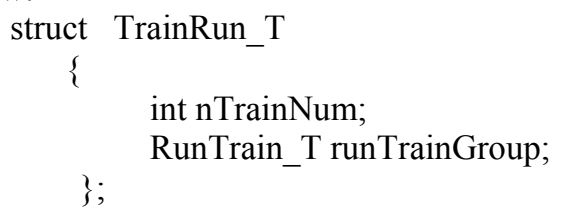

Firstly, the federate-TrainRun publishes its object class to the HLA platform. Then it will send simulation data to HLA. If one federate needs the data, it should firstly subscribe the object class-TrainRun, and then receives the data from the federate-TrainRun. The progress of simulation data exchange is shown in Fig. 4.

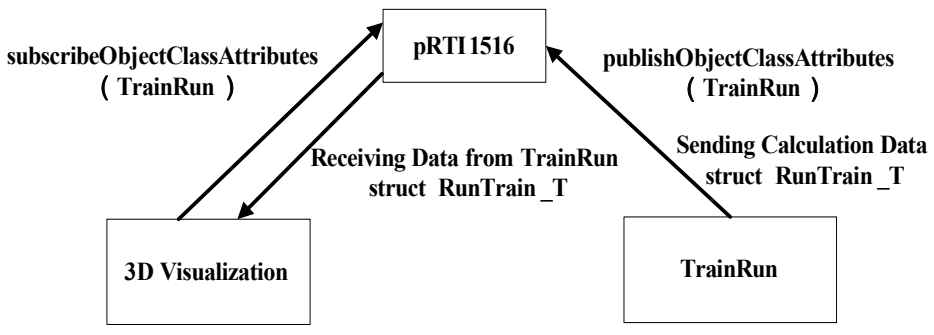

Figure 4: Progress of data exchange. 


\subsection{Time Advance of HLA}

pRTI1516 provides the time advance mechanism of HLA. For every federate in the whole federation, there are two ways of time advance. One is independent time advance and the other is consulting time advance. As for every federate, they have different simulation time steps. In order to keep synchronization of data exchange between two federates, the method-child node controlled is adopted. In every simulation cycle, it will apply for time advance through HLA thread when the federate that calculates faster finishes its one step simulation. The application will continue until the time advance authority is obtained. After the last federate finishes its time advance application, the time management service of HLA makes the whole federation advance. As Shown in Fig. 5.

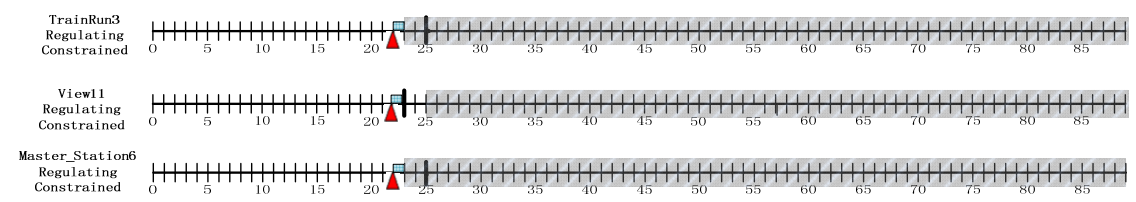

Figure 5: Time advance of simulation (partial modules).

\subsection{Simulation Progress of pRTI}

Every federate runs in one computer of the local area network while simulation. After the initializing data of every federate is set up, the administrator can choose to give a signal or not such as simulation start, simulation end. In this paper, these communication control orders are sent by the master station.

\section{Main simulation modules}

\subsection{On-board CCU}

On-board CCU (centre control unit) is in charge of the logic control, traction/braking force's allocation and collection and the processing of status data/fault data of the whole train. CCU is a very important part of the high speed railway train. On-board CCU receives operation orders from drivers and on-board ATP. The train traction force and braking force will then be redistributed through logic control. Thus the train's safety will be guaranteed.

\subsection{Driver control device}

As the simulation operation machine, driver control device (DCD) is an important interface between users and machine. Shown in Fig. 6, drivers can operate the buttons and handles to send orders to other federate in the simulation platform. As stated in the abstract, DCD offers the interface of train's auxiliary operation system. The train's auxiliary operation system is tested during the simulation. 


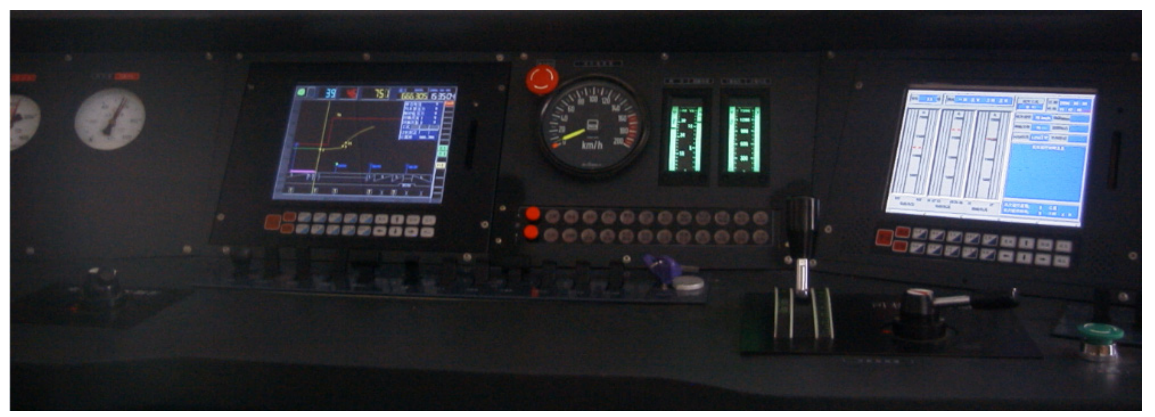

Figure 6: Driver control device.

\subsection{Signal system and power supply system}

The signal simulation module (Fig. 7), the traction power supply simulation module and the train operation simulation module together simulate the running of an actual train. The train's auxiliary operation module calculates out instructional speed profiles and switching points according to the train's running working conditions and then shows corresponding orders according to different working conditions and handling levels. Finally, the distributed simulation platform is proved to be efficient through the test of the train's auxiliary operation module.

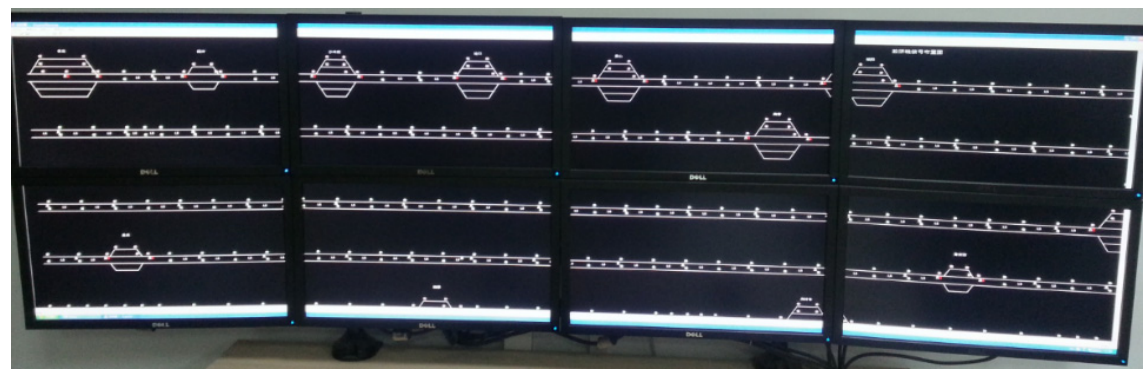

Figure 7: Signal system.

\section{Simulation}

In order to verify the distributed simulation platform, the united simulation is a necessity. Take JingJin intercity railway line as an example. The experiment train is $\mathrm{CRH} 2 \mathrm{C} 350$, and its basic simulation parameter is as follows. 
Table 1: Train's basic parameters.

\begin{tabular}{|c|c|c|c|c|c|}
\hline $\begin{array}{c}\text { Train } \\
\text { Marshalling }\end{array}$ & $\begin{array}{c}\text { Train } \\
\text { Capacity }\end{array}$ & $\begin{array}{c}\text { Train } \\
\text { Weight }\end{array}$ & $\begin{array}{c}\text { Train } \\
\text { Length }\end{array}$ & Max Speed & $\begin{array}{c}\text { Traction } \\
\text { Power }\end{array}$ \\
\hline $\begin{array}{c}6 \text { motor cars }, \\
2 \text { trailer cars }\end{array}$ & 610 persons & $420.4 \mathrm{t}$ & $201.4 \mathrm{~m}$ & $350 \mathrm{~km} / \mathrm{h}$ & $7342 \mathrm{~kW}$ \\
\hline
\end{tabular}

The federate-TrainRun calculates out the basic information of the train operation, and the results will be sent to the display interface through HLA. Then View subscribes TrainRun and receives train running data. Fig. 8 is the simulation result comparison. The solid line is the actual experiment profile in Jingin Line, 2008. The dotted line is the simulation profile after the TAOS is introduced.

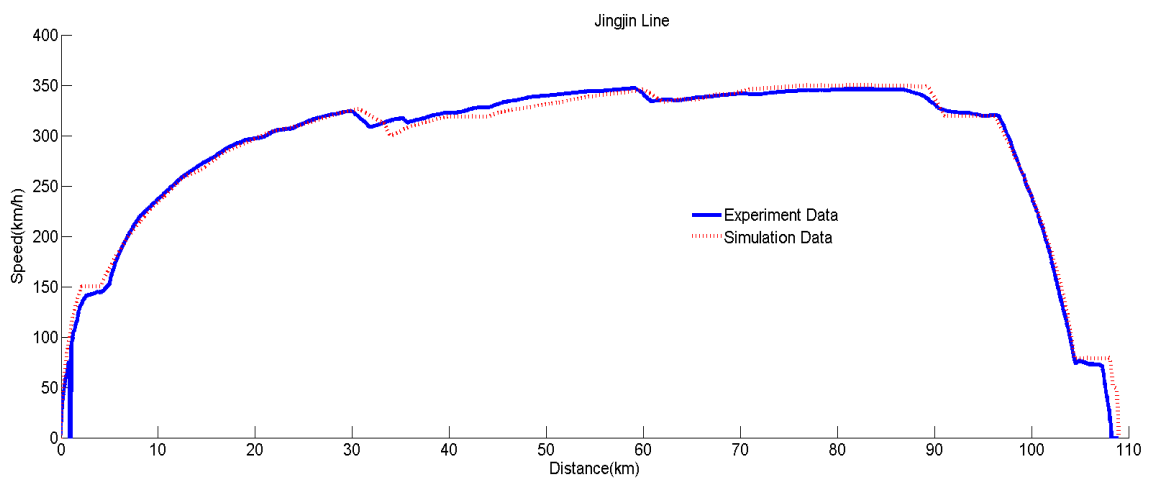

Figure 8: Jingjin line data comparison.

Obviously, the two profiles are nearly the same because the TrainRun module is designed according to the traction calculation rule in China. Meanwhile, the driving experience of excellent drivers is also introduced in the TAOS that give the drivers dynamic driving orders when train is running.

However, deviation still exists between two profiles. There are two main reasons. Firstly, the basic resistance formula of train operation is different from that when the train is running on the actual line. Due to influence of the wind speed and wind direction on the railway line, the resistance force is always changing. So the deviation exists during the operation of a train. Secondly, different drivers can't give absolutely the same handle levels to the train because of their driving habits and the driving environment where they stay in.

\section{Conclusion}

This paper introduces the implementation and design of the high-speed train's running simulation platform based on HLA. At the same time, the train's auxiliary operation system is tested and verified as well. The test result shows that the 
simulation platform is efficient and the train's auxiliary operation system can give instructional orders to the driver when the train is running. The auxiliary operation system still needs to be improved to make the distributed simulation platform perform better, such as improving the online control algorithm of the auxiliary operation system.

\section{References}

[1] J. Lu, B. Ning. The Design and Implementation of a Train's 3D Driving Simulation System. Railway Signalling \& Communication, 2008: 37-40.

[2] X. W. Song, T. Tang. Application of Scene Visual Simulation in Subway Trains Operation Control System. Journal of Beijing Jiaotong University, 2007: 67-71.

[3] H. Su, M. Y. Zhou. Distributed Visual Simulation System in Train Simulator. Journal of China Railway Society, 2002: 37-41.

[4] H. Pu, W. Li, A. X. Long. Study on Traction Calculation of High-speed Railway and 3D Running Simulation. Journal of Railway Science and Engineering, 2011: 1-5.

[5] C. L. Song, Y. Zhang. Implementation and Design on Train Movement Simulator of Distributed Three-Dimensional Visualization. Railway Computer Application, 2008: 27-30.

[6] L. You, Brief Introduction of HLA. Southwest Jiaotong University, 2011.

[7] Pitch pRTI run-time infrastructure. http://www.hwacreate.com.cn/cn.

[8] J. L. Zhu, H. C. Li, Q. Y. Wang, et al. Optimization Analysis on the Energy Saving Control for Trains. China Railway Science, 2008, 29(2): 104-108. 